\title{
Técnica de los osteotómos en implantología
}

\author{
Vilaplana Gómez J.A.* \\ Méndez Trujillo $\mathrm{S} . * *$ \\ Ortega López J.J.*** \\ Vilaplana Vivo $\mathrm{J} . * * *$
}

Vilaplana Gómez J.A.; Méndez Trujillo S.; Ortega López J.J.; Vilaplana Vivo J.; Técnica de los osteotomos en implantología, 2000; 12

\begin{abstract}
RESUMEN
La ausencia de dientes puede crear situaciones de extrema reabsorción ósea, impidiendo en ocasiones la inserción de implantes debido al limitado volumen del hueso alveolar residual. En estos casos, la técnica de los osteotomos puede permitirnos separar y comprimir el hueso, creando un lecho artificial donde insertar los implantes.

En el presente artículo presentamos una serie de 18 pacientes, en los que se insertaron 40 implantes Steri-oss cilíndricos recubiertos de hidroxiapatita, en el periodo comprendido entre septiembre del 1995 y febrero de 1998. Se describe la técnica y se presentan los resultados obtenidos.

Durante la intervención quirúrgica no surgieron complicaciones significativas, ni tampoco en el postoperatorio inmediato. Después de la segunda intervención quirúrgica, y antes de iniciar la fase protésica, dos implantes de diferentes pacientes mostraron signos de ausencia de osteointegración, procediéndose a su retirada.

En nuestra opinión, la técnica de los osteotomos creemos que puede ser una buena solución en aquellos casos en los que la anchura del hueso alveolar no nos permita la inserción de implantes.
\end{abstract}

\section{PALABRAS CLAVE}

Implantes. Osteointegración. Osteotomos.

\section{INTRODUCCIÓN}

El mantenimiento de los rebordes alveolares edéntulos tras la pérdida de los dientes, ha supuesto un constante problema durante muchos años. Los métodos clásicos de rehabilitación preprotésica se han basado en la colocación de injertos de diverso origen, como el hueso autógeno (1), o sustitutos óseos inertes, como la hidroxiapatita reabsorbible unida a partículas de hueso corticoesponjoso autógeno, con el fin de conseguir una estructura que retrase el proceso de reabsorción (2). Estas técnicas presentan muchas dificultades para el mantenimiento del material de injerto y para su recubrimiento.

El hueso alveolar de la premaxila se remodela rápidamente tras la pérdida de los dientes naturales. La anchura de la cresta puede disminuir hasta en un 40$60 \%$ durante los tres primeros años que siguen a la pérdida dental (3). La cresta anterior del maxilar superior, que originalmente tiene un espesor de unos $8 \mathrm{~mm}$, puede reabsorberse llegando a tener en algunos casos menos de $3 \mathrm{~mm}$ al cabo de cinco años (4). La atrofia del proceso alveolar o la presencia de concavidades vestibulares, pueden limitar y dificultar la inserción de implantes. Para la remodelación ósea, es muy importante mantener intacta las células del estrato interno de la cubierta perióstica alveolar labial en las extracciones dentales (5). El hueso cortical recibe del periostio un $80 \%$ de su aporte sanguíneo y drena en el mismo el $100 \%$ del retorno venoso. (6)

La reabsorción ósea del maxilar superior a supuesto un gran reto para los especialistas. Para su tratamiento disponemos de diversas técnicas, como injertos óseos, injertos unidos a técnicas de regeneración e injertos subantrales de los senos maxilares.

\footnotetext{
* Profesor Asociado de Cirugía. Facultad de Odontología. Universidad de Murcia

** Jefe del Servicio de Cirugía Maxilofacial H. Virgen de la Arrixaca. Profesor Asociado de Cirugía Facultad de Odontología. Universidad de Murcia

*** Profesor colaborador de cirugía
} 
En los ochenta se empiezan a describir procedimientos de expansión ósea. En 1985 Osbord (7), presenta un método que consistente en movilizar un segmento óseo vestibular en su totalidad. En 1986 Nentwig (8) describe el método conocido como "bone-splitting", según el cual el proceso alveolar se escinde empleando un cincel, método también empleado por Frisch y Jacobs (9). Scipioni (10) realizo un estudio prospectivo sobre 170 pacientes a quienes se les insertaron 329 implantes observando un $98,7 \%$ de éxitos tras un periodo de cinco años de seguimiento. Fue Tatum (11) el que a finales de los ochenta, comenzó a experimentar con técnicas de expansión. Pero ha sido Summers $(12,15)$, el que con más acierto ha diseñado unos instrumentos conocidos como osteotomos con los que se ha ido desarrollando y perfeccionando la técnica hasta nuestros días. Estos instrumentos son de forma cilíndro-cónica y, van aumentando de grosor progresivamente, para expandir y compactar las trabéculas óseas. De esta forma se va aumentando la densidad del hueso y la superficie de fricción. Los osteotomos de punta fina (16) los utilizamos en aquellos casos en que nos encontramos un hueso tipo II o III en los que no necesitamos conseguir una compactación sino una expansión debido a la estrechez ósea, como son las crestas en "filo de cuchillo". La configuración más punzante de los osteotomos nos permitirá expandir las crestas de forma más controlada.

En los casos que presentamos hemos utilizado una técnica mixta, consistente en el empleo de osteotomos en las zonas más estrechas y fresas donde la cresta se va ensanchando, con el fin de conseguir la mayor superficie de osteointegración. El objetivo de este trabajo es exponer nuestra experiencia en la técnica de expansión de la cresta alveolar en el maxilar superior, siguiendo el método descrito por Summers.

\section{MATERIAL Y MÉTODO}

Realizamos un estudio prospectivo en el que fueron incluidos 18 pacientes, en los que se indicó la inserción de 40 implantes cilíndricos Steri-oss recubiertos de hidroxiapatita; en cinco casos se le insertó un solo implante, en siete casos dos, en tres casos tres y en otros tres casos cuatro implantes.

De los 18 pacientes, el 33\% eran varones y $66 \%$ mujeres. La edad media es de 37 años (rango de 16 a 58). Los dividimos en dos grupos: pacientes de edad hasta 25 años, que recibieron 16 implantes, y pacientes de más de 25 años, con 34 implantes insertados.

La colocación de los implantes tuvo lugar durante el periodo comprendido entre septiembre de 1995 y febrero de 1998 (periodo comprendido de 18 a 60 meses).
El motivo de la ausencia dental fue: en 8 casos por agenesia, en 20 por caries dental, en 1 caso por extracción traumática con perdida del hueso alveolar, en 2 casos por luxación con perdida dental por tratamiento ortodóntico incorrecto, en 8 casos por accidente y en 1 caso por quiste.

Los dientes repuestos se agruparon en tres grupos: 30 implantes fueron insertados en el sector anterior (incisivos y caninos), 6 en el sector posterior (premolares y molares) y 4 en un desdentado total. Todos los casos corresponden al maxilar superior.

La técnica de utilización de los osteotomos no varió en ningún caso. La única diferencia consistió en la colocación de injerto óseo, que fue necesario en 20 de los implantes. En estos casos, se les reforzó el alvéolo vestibular con injerto óseo autógeno 50\%, mezclado con hueso desmineralizado liofilizado $30 \%$ e hidroxiapatita microporosa reabsorbible $20 \%$.

El tamaño de los implantes colocados fue: de 3,25x12 $\mathrm{mm}$ se insertaron 23 , de $3,25 \times 14 \mathrm{~mm}, 13$ y de $3,8 \times 12$ $\mathrm{mm}, 4$. En todos los casos, los implantes eran cilíndricos de superficie rugosa recubiertos de hidroxiapatita. Todos los casos se intervinieron con anestesia local más sedación, excepto en dos, en los que se le aplicó anestesia general porque además se realizó una elevación de seno.

\section{TÉCNICA QUIRÚRGICA}

En todos los casos realizamos la expansión del proceso alveolar siguiendo la técnica descrita por Summers (12-15). Realizamos un fino orificio central en el reborde de la cresta ósea y procedemos a ensancharla con el osteotomo (Fig. 1) más estrecho (Fig. 2) hasta alcanzar la profundidad del implante que vamos a insertar;

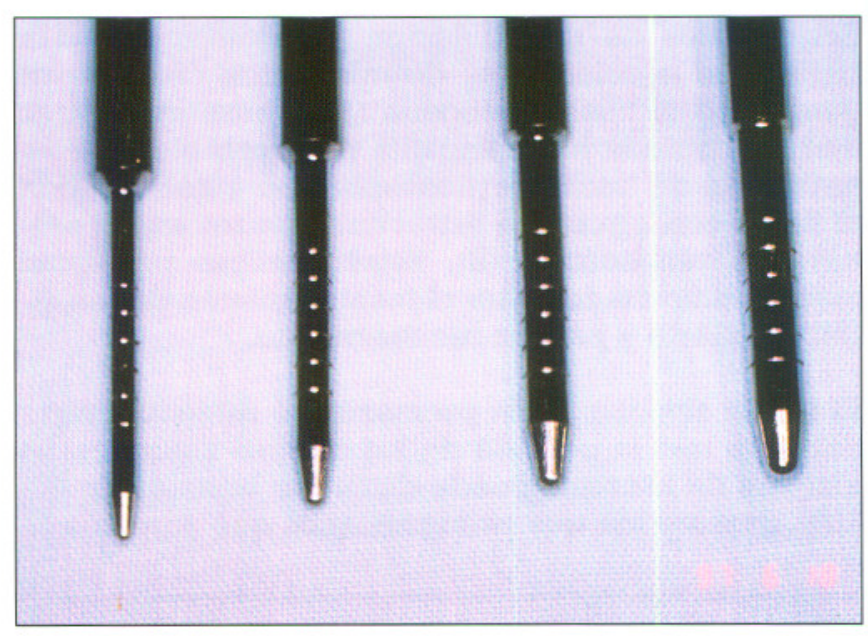

Fig. 1. Técnica de los Osteotómos 


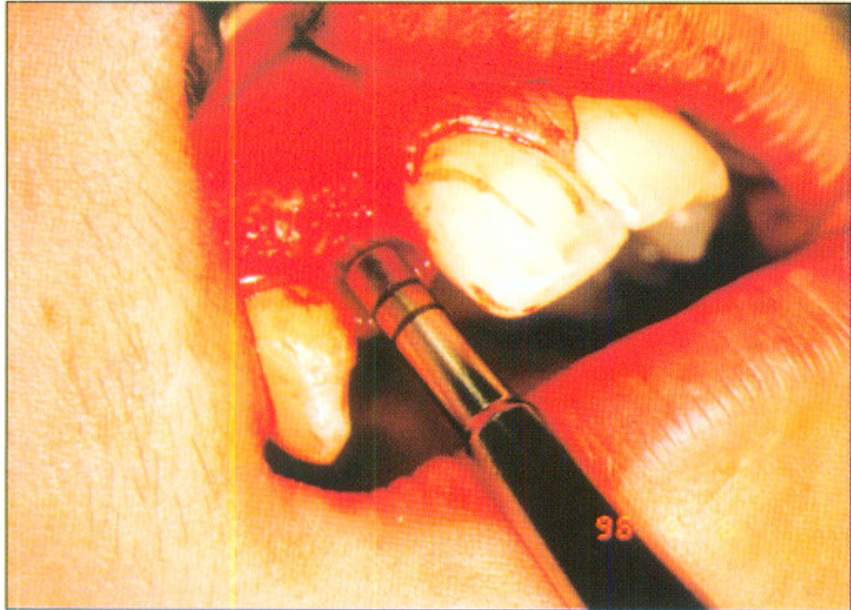

Fig. 2. Técnica de los Osteotómos

progresivamente se va aumentando ensanchando hasta alcanzar el diámetro final deseado (Fig. 3). Para el paciente resulta considerablemente más cómodo la introducción del instrumento presionando con la mano, pero cuando esto no es posible, recurrimos a percutir suavemente con un martillo. A continuación realizamos movimientos giratorios cuidadosamente calculados de presión y expansión.

El instrumental giratorio solo lo empleamos cuando el grosor del hueso lo permite; en caso contrario no necesitaremos irrigar ya que apenas se produce calentamiento. En los casos en que las crestas son muy estrechas, pero con hueso muy denso, utilizamos osteotomos con la punta afilada (16) en vez de cóncava, realizando giros sin percutir.

Cuando tenemos el lecho artificial preparado, insertamos él implante, siempre de un grosor ligeramente superior(Fig. 4).

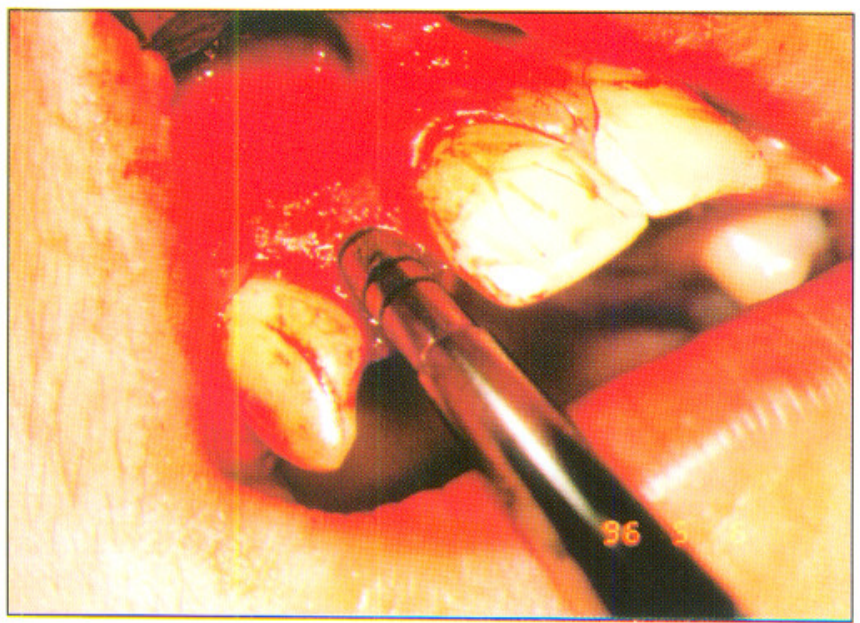

Fig. 3. Técnica de los Osteotómos

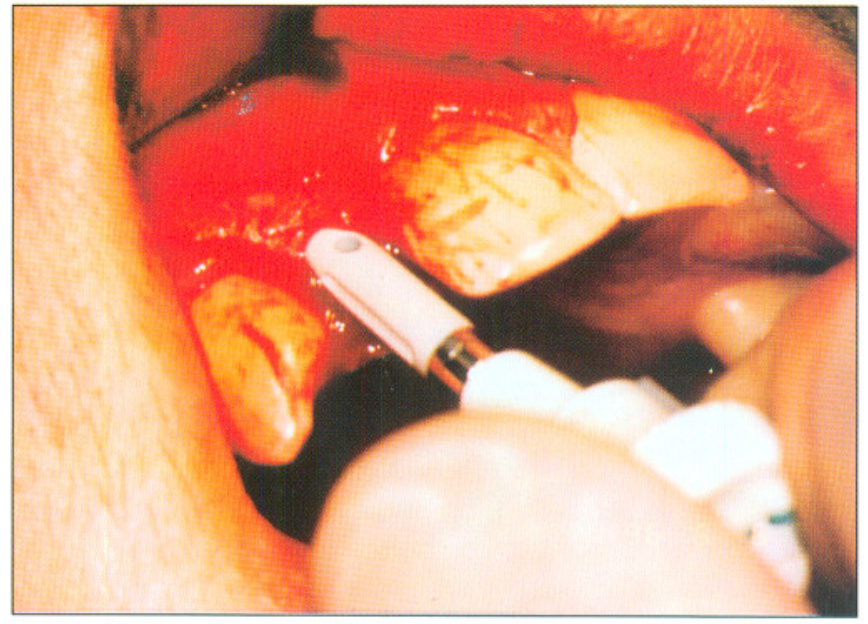

Fig. 4. Técnica de los Osteotómos

Todos los pacientes fueron revisados a la semana, quince días, un mes, tres y seis meses. Ningún caso presentó complicaciones.

\section{RESULTADOS}

El postoperatorio inmediato y el seguimiento posterior que se realizó a los pacientes transcurrió sin ninguna complicación. A los seis meses después de su colocación, los implantes estaban clínicamente estables y osteointegrados. No existía alteración periimplantaria en ningún caso.

En la segunda fase quirúrgica, se colocaron los pilares de cicatrización y al mes, cuando iniciamos la fase protésica, dos de los cuarenta implantes colocados presentaron signos de ausencia de osteointegración. No hubo rechazo pero su estabilidad no era totalmente correcta, procediendo a su retirada.

Los dos casos de pérdida del implante no correspondían al mismo paciente, y en ambos casos los otros implantes colocados no presentaron complicaciones. Se perdieron, un incisivo lateral superior derecho y un segundo premolar superior izquierdo. La característica fundamental en ambos casos fue que en la fase quirúrgica de creación del lecho artificial, se produjeron microfracturas en la tabla ósea alveolar vestibular (17), que es la que sufre más intensamente los efectos de la expansión ósea. En los dos casos, cubrimos el defecto con hueso obtenido de áreas vecinas mezclado con hueso liofilizado e hidroxiapatita y colocamos una membrana reabsorbible de colágeno. No presentaron alteraciones clínicas ni radiológicas hasta el momento de recibir la carga. Pensamos que en los casos en que producen fracturas longitudinales y colocamos injertos, el período de osteointegración deberá ser más largo (Fig. 5-8). 


\section{AVANCES}

Volumen 12 - $\mathrm{N}^{\circ} 1$ - Mayo 2000

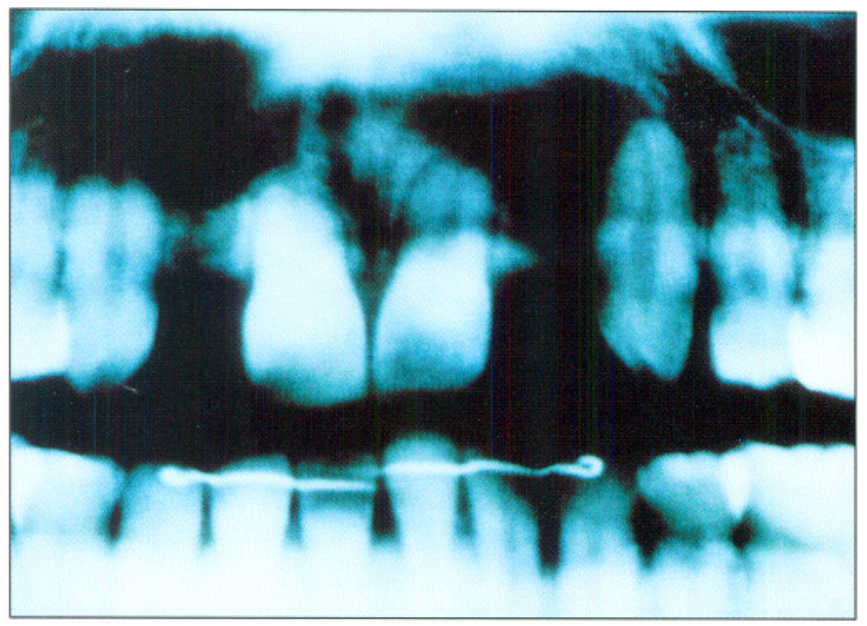

Fig. 5. Técnica de los Osteotómos

\section{DISCUSIÓN}

La técnica de los osteotomos de Summers ha significado una excelente solución en aquellos casos en que la estrechez ósea alveolar no nos permitía insertar implantes, teniendo que recurrir a procedimientos de regeneración ósea que resultaban complicados e incómodos para el paciente.

En las clases D3 y D4 de Misch (18), con el instrumental descrito conseguimos una mayor condensación de la esponjosa, aumentando la superficie de fricción primaria con él implante. La mayor superficie de osteintegración, le proporciona una estabilidad mucho mejor al implante insertado.

Hasta que surgen las técnicas de expansión alveolar, la fijación de los implantes que conseguíamos en todos los procesos de regeneración ósea, no nos pro-

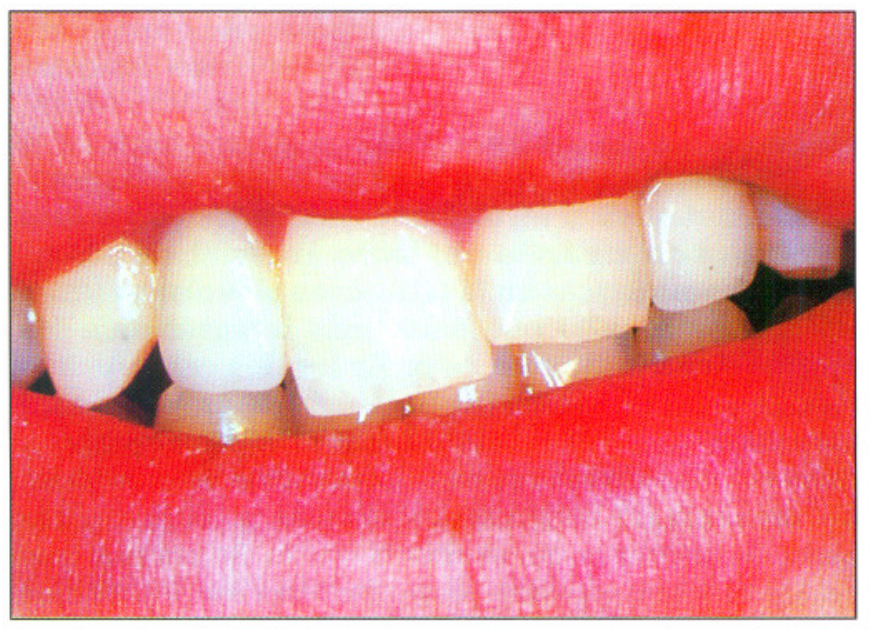

Fig. 6. Técnica de los Osteotómos

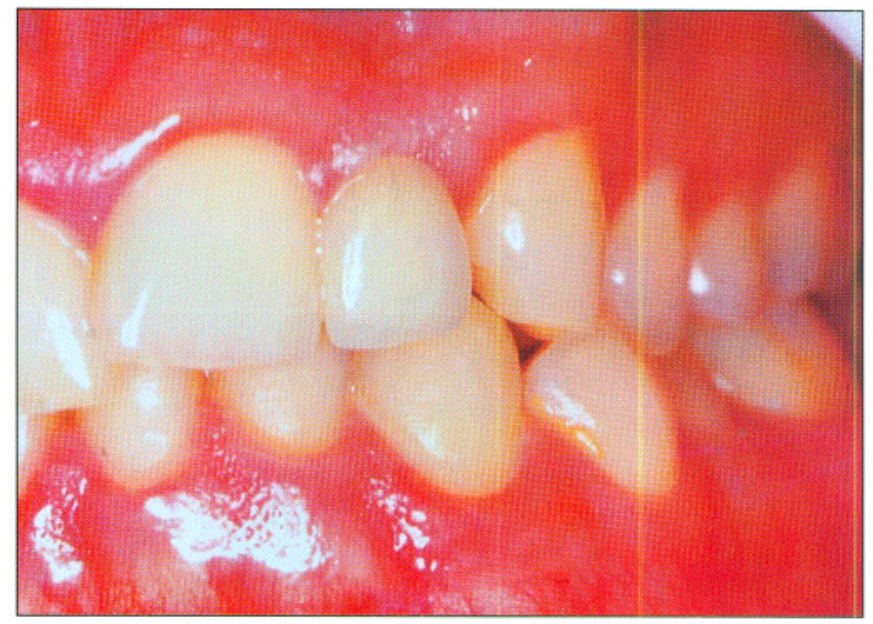

Fig. 7. Técnica de los Osteotómos

porcionaba los mismos resultados y al paciente le resultaban largos y molestos.

Thomas (20) sugiere que los biomateriales que recubren la superficie del implante juegan un papel importante en la osteointegración, y según Janesen (21) el recubrimiento con hidroxiapatita proporciona pequeños puentes entre el implante y el diente.

En el trabajo que nosotros presentamos todos los implantes llevaban recubrimiento de hidroxiapatita en su superficie, obteniendo unos resultados muy satisfactorios. Pero no podemos hacer valoraciones por falta de estudios comparativos.

En todos los pacientes que presentamos en el estudio se consiguió una correcta rehabilitación protésica, mostrando su satisfacción con el tratamiento realizado y el resultado obtenido.

\section{CONCLUSIONES}

- La técnica de expansión ósea con osteotomos nos permite insertar implantes en crestas alveolares estrechas ya que mantenemos la cresta ósea alveolar y no perdemos sustrato óseo.

- Al compactar el hueso esponjoso incrementamos la fricción.

- Cuando la resistencia ósea es mayor, utilizamos fresas además de los osteotomos.

- Si la densidad ósea es pequeña, para que él implante quede bien impactado, insertaremos uno de mayor grosor.

- El implante se colocara inmediatamente, para evitar que las tablas óseas se cierren y nos dificulte la introducción.

- En caso de existir concavidades en el alvéolo vestibular evitamos perforaciones. 


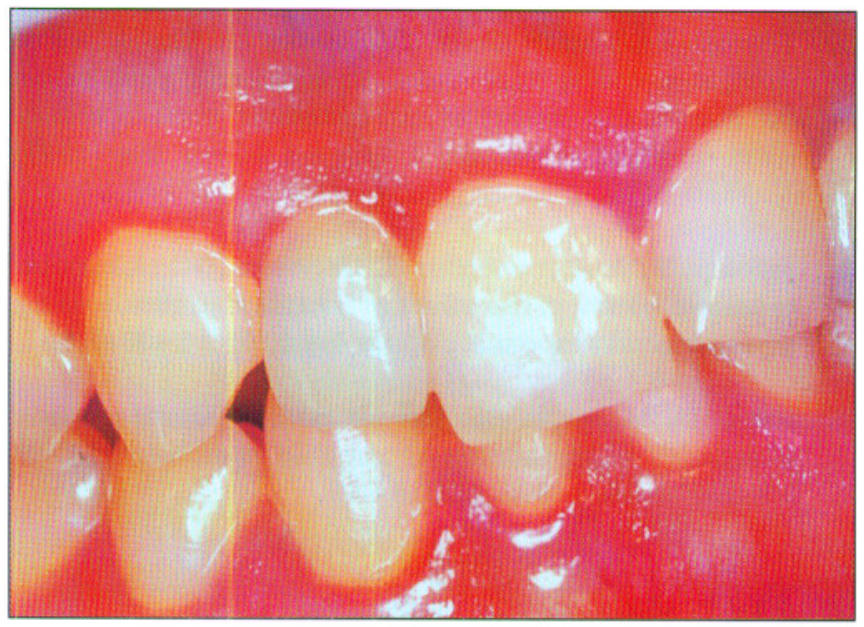

Fig. 8. Técnica de los Osteotómos

\section{SUMMARY}

The absence of teeth creates situations of extreme bone resorption, not allowing the installation of dental implants due to narrowness of the residual alveolar bone. In these cases, the osteotome technique permits us to separate and compress the bone, creating an proper artificial bed for the implants insertion.

In the present article we present a series of 18 patient, in whom 40 Steri-oss cylindrical HA-coated implants were installed; implants placement took place from September 1995 to February 1998. We describe the technique and present the results.

During the surgical procedure no complications were observed, neither during the postoperative period. After the second stage surgery, and before the starting of the prosthetic procedures, two implants corresponding to two different patients exhibit signs of absence of osseointegration, and we proceed to remove them.

We believe that the osteotomes technique is a good solution for those patients in which the width of the alveolar bone was not adequate for the implants installation.

\section{KEY WORDS}

Implants. Osseointegration. Osteotomes.

\section{CORRESPONDENCIA:}

\section{Dr. J.A. Vilaplana Gómez}

Facultad de Odontología. Universidad de Murcia

\section{BIBLIOGRAFÍA}

1. BOYNE PJ.: Preprosthetic surgery for the older patient. J Am Dent Assoc 1987 (spec issue): Jan-Feb, p 17.

2. Boyne PJ.: The use of marrow particulate grafts and titanium implants in the reconstruction of resected mandibles, in Jacobs JR (ed): Maxillofacial Trauma: An International Perspective, vol 19. Praeger, 1983, pp 212218.

3. Pietrovski J. The bony residual ridge in man. J Prosthet Dent 34: 456-462- 1975.

4. Tatun OH.: The Omni implantsystem, abstract, Alabama Implant Congres, May 7, 1987.

5. Roberts EW.: Bone phisiology and metabolism, Can Dent Assoc J 75:54-6 1987.

6. Chanavaz M.: Blood supply to the cortical bone, abstract, Alabama Impant Study Group, Birmingham, Alabama, May, 1991.

7. Osborn JF.Die Alveolar-Extensionplastik. Teil I u. II. Quintessenz 1985; 36: 9, 239.

8. Nentwig GH. Die Technik des Bone Splitting dei alevolären Rezessionen im Oberkiefer-Front-bereich. Quintessenz 1986; 35: 1.825.

9. Frisch E. Jacobs HG. Krüger J Engelke W.: Bone Spliting als unterstützende Manahme beizahnärztlich-chirurgischen Eingriffen. Z Zahnärtzl Implantol 1992;7:122-126.

10. Scipioni A. Bruschi G. Calesini G.:The edentulous ridge expansion technique: $A$ five year study. Int J Periodont Rest. 14: 451-459. 1994.

11. Tatum H. Mxillary and sinus implant reconstructions. Dent. Clin. Of North Am., 1986; 30: 207-229.

12. Summers RB. A new concept in maxillary implant surgery: the osteotome technique. Compendium $1994 \mathrm{Feb}$; 15(2):152.

13. Summers RB. The osteotome technique:Part2-The ridge expansion osteotomy (REO) procedure. Compendium 1994 Apr;15(4):422.

14. Summers RB.: The osteotome technique: Part 3-Less invasive methods of elevating the sinus floor. Compendium 1994 Jun;15(6):698.

15. Summers RB. The osteotomy technique: Part4-Future site development. Compend Contin Educ Dent 1995 Nov; 16(11):1080. 
16. Anitua E. Ensanchamiento de cresta en el maxilar superior para la colocación de implantes: técnica de los osteotomos. Actual. Implantol., 1995;7:65-72.

17. Wachtel H.C. Langord A.Bernimoulin J.P. Reichard P.: Guided bone regeneration next of osseointegrated implants in humans. Int. J oral max.-fac Implants 6:127135.1991.

18. Misch CE.: Divisions of available bone in implant dentistry. Int J Oral Implantol. 1990;7-9.

19. Steflik D.E. Lacefield W.R. Sisk A.L. Parr G.R. Lake F.T: Patterson J:W.: Implantes dentales revestidos con hidroxiapatita: histología descriptiva e histomorfometría cuantitativa. J Oral Impl., Septiembre 1994.
20. Thomas KA. Kay JF. Cook SSD. Jarcho M.: The effect of surface macro texture and hydroxylapatite profiles on the mechanical strengths and histologic profiles of titanium implant materials. J Biomed Mater Res 1987; 32:1395-1414.

21. Janesen JA. Vanderwaerden JPCM. Woke JGC.: A histological evaluation of the effect of Hydroxylapatite coating on interfacial response. J Mater Med 1993;4:466-470.

22. Hupp JR. McKenna SJ.: Use of porous hidroxyapatite blocks for augmentation of atrophic mandibles. J Oral Maxillofac Surg 1998; 46:538-545. 